[2] He, Y., et al., SIRT6 inhibits TNF-alpha-induced inflammation of vascular adventitial fibroblasts through ROS and Akt signaling pathway. Exp Cell Res, 2017. 357(1): p. 88-97.

[3] Wei, Y., et al., Inhibitory Effect of a Novel Antirheumatic Drug T-614 on the IL-6-Induced RANKL/OPG, IL-17, and MMP-3 Expression in Synovial Fibroblasts from Rheumatoid Arthritis Patients. Biomed Res Int, 2015. 2015: p. 214683.
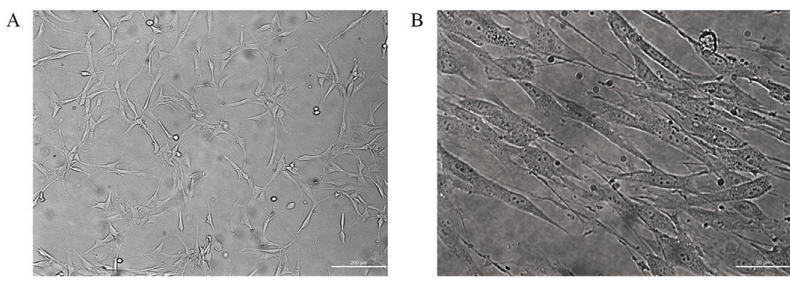

Figure 1.Clutured human aortic adventitial fibroblasts(HAAF) in vitro. HAAF were polygonal or spindle-shaped under the microscope. (A) Appearance of HAAF at generation 5, x100; (B) Appearance of HAAF at generation $5, x 400$

Abstract THU0024 Figure 1

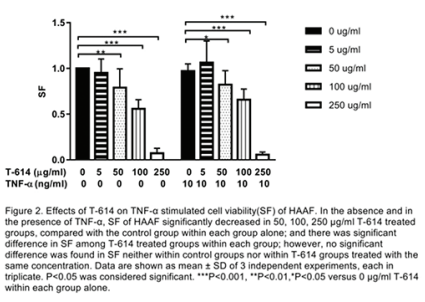

Abstract THU0024 Figure 2 .

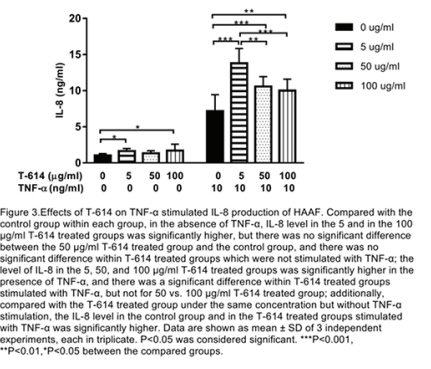

Abstract THU0025 Figure 2

Acknowledgement: Funding: This project was supported by grants from China International Medical Foundation(Z-2014-06-2-1636). The sponsors had no role in the study design, data collection and analysis, decision to publish, or preparation. Disclosure of Interests: None declared

DOI: 10.1136/annrheumdis-2019-eular.354

\section{THU0025 ANALYSIS OF POTENTIAL INTERACTIONS BETWEEN TENOCYTES AND SYNOVIAL FIBROBLASTS AFTER STIMULATION WITH CYTOKINES EXPRESSED WITHIN THE SYNOVIO-ENTHESAL COMPLEX}

Felix Dechant ${ }^{1}$, Klaus Frommer ${ }^{1}$, Neal L Millar ${ }^{2}$, lain Mcinnes $^{2}$, Stefan Rehart ${ }^{3}$, Ulf Müller-Ladner ${ }^{1}$, Elena Neumann' ${ }^{1}{ }^{1}$ Justus-Liebig-University Giessen, Dept. of Rheumatology and Clinical Immunology, Bad-Nauheim, Germany; ${ }^{2}$ University of Glasgow, Inst. of Infection Immunity and Inflammation, Glasgow, United Kingdom; ${ }^{3}$ Agaplesion Markus Hospital, Dept. of Orthopaedics and Trauma Surgery, Frankfurt, Germany

Background: Psoriatic arthritis (PSA) is frequently associated with enthesitis. It has been proposed that inflammatory processes at the synovioenthesal complex are involved in the pathogenesis of inflammatory arthritides including especially PsA. Besides IL-1 $\beta$ and TNF- $\alpha$, IL-15, IL-23 and IFN- $\gamma$ are cytokines expressed within the synovium, tendons and entheses and some of which are already used as therapeutic targets. While synovial fibroblasts (SF) are known key effector cells of cartilage destruction in inflammatory arthritides such as RA, tenocytes are a major component of tendons and entheses and play a central role in tendon inflammation observed in PsA.

Objectives: To investigate whether PSASF and tenocytes show significant interactions while being stimulated with the above cytokines alone as well as in combination with the aim to find out whether these may contribute to the pathogenesis of PsA.

Methods: SF were isolated from patients with PsA undergoing joint surgery. Human tenocytes were acquired commercially and isolated from hamstring tendon tissue of patients undergoing hamstring tendon $\mathrm{ACL}$ reconstruction. PSASF and tenocytes were stimulated with IL-1 $\beta$, TNF- $\alpha$ IFN- $\gamma, \mathrm{IL}-15$ and IL-23 alone and in combination. Direct cell co-culture experiments were performed at a 1:1 ratio of both cell types in paralle to experiments with single cell type cultures. IL-6 levels were measured by ELISA to quantify the immunological activation of the cells.

Results: PSASF as well as tenocytes showed strong responses to IL-1 $\beta$ (tenocytes $\uparrow 173$-fold, $\mathrm{n}=3$; PSASF $\uparrow 56$-fold, $\mathrm{n}=3$ ) and TNF- $\alpha$ (tenocytes $\uparrow 10$-fold, $\mathrm{n}=3$; PSASF $\uparrow 9$-fold, $\mathrm{n}=3$ ) stimulation regarding $\mathrm{IL}-6$ secretion. IFN- $\gamma$ alone had only minimal effects on both cell types but acted synergistically when applied together with $\mathrm{IL}-1 \beta$ (tenocytes $\uparrow 218$-fold, $\mathrm{n}=3$; PsASF $\uparrow 129$-fold, $n=3$ ) and TNF- $\alpha$ (tenocytes $\uparrow 24$-fold, $n=3$; PsASF $\uparrow 19$ fold, $\mathrm{n}=3)$. IL-15 and IL-23 alone showed no effect but the data suggest a small antagonistic effect against IL- $1 \beta$ (tenocytes IL-15 $\downarrow 21 \% / \mathrm{LL}-23$ $\downarrow 27 \%, n=3$; PsASF IL-23 $\downarrow 19 \%, n=3)$ and TNF- $\alpha$ induced IL- 6 secretion. Overall, PsASF and tenocytes showed similar responses in the single cell type stimulation experiments. Co-culturing PsASF and tenocytes did not reveal any synergistic or antagonistic interactions in regards to any of the cytokines used.

Conclusion: Our data suggest that tenocytes and PsASF do not interact in a way that would promote inflammation within the synovio-enthesal complex. Also, as far as the induction of IL-6 is concerned, PsASF and tenocytes are not major target cells of IL-15 and IL-23. IFN- $\gamma$, however may be able to promote inflammation in combination with other cytokines in both cell types.

Disclosure of Interests: Felix Dechant: None declared, Klaus Frommer: None declared, Neal L Millar: None declared, lain McInnes Grant/research support from: AstraZeneca, Celgene, Compugen, Novartis, Roche, UCB Pharma, Consultant for: AbbVie, Celgene, Galvani, Lilly, Novartis, Pfizer UCB Pharma, Stefan Rehart: None declared, Ulf Müller-Ladner Grant research support from: Projekt supported by an unrestricted educational grant from Celgene $\mathrm{GmbH}$., Elena Neumann: None declared

DOI: 10.1136/annrheumdis-2019-eular.7148

\section{THU0026 TARGETING SYNOVIAL FIBROBLASTS IN RHEUMATOID ARTHRITIS BY PEFICITINIB AND FILGOTINIB}

Magnus Diller ${ }^{1}$, Rebecca Hasseli ${ }^{1}$, Iris Aykara ${ }^{1}$, Marie Hülser ${ }^{1}$, Stefan Rehart ${ }^{2}$, Ulf Müller-Ladner ${ }^{1}$, Elena Neumann ${ }^{1} .{ }^{1}$ Campus Kerckhoff, Justus-Liebig-University Gießen, Dept. of Rheumatology and Clinical Immunology, Bad Nauheim, Germany, ${ }^{2}$ Agaplesion Markus Hospital, Dept. of Orthopaedics and Trauma Surgery, Frankfurt, Germany

Background: The Janus kinase inhibitors (JAKi) peficitinib and filgotinib are currently examined in clinical trials for treatment of rheumatoid arthritis. Both inhibitors are well tolerated up to doses causing Cmax values higher than $1 \mu \mathrm{M}[1,2]$. This is in contrast to the approved dosages of tofacitinib and baricitinib reaching $\mathrm{Cmax}$ values below $0.5 \mu \mathrm{M}[3,4]$. How ever, it is not known if the higher concentrations of peficitinib or filgotinib offer a benefit in treatment of rheumatoid arthritis.

Objectives: The aim of the study compared the effect of different JAKi on inflammatory response and functional behavior of fibroblast-like synoviocytes from patients with RA (RASF).

Methods: Human RASF were isolated and pretreated with JAKi. After stimulation with IL-1 $\beta$ and JAKi with/without soluble IL-6 receptor (sIL-6R) the levels of IL- 6 and MMP-3 were measured in supernatants by ELISA. The effect of different JAKi on proliferation of RASF was determined by a BrdU-incorporation assay. The influence of peficitinib on migration of RASF towards a FCS gradient was examined. For short-term adhesion assays, cells were treated with JAKi, detached and seeded in culture plates. The plates were extensively shaken and adherent RASF quantified by counting crystal violet stained cells. Cell viability, cytotoxicity and apoptosis were measured using commercially available assays.

Results: The IL-1 $\beta(10 \mathrm{ng} / \mathrm{ml})$ dependant IL-6 release of RASF was decreased by peficitinib $(62 \%, p<0.001)$ and by filgotinib $(30 \%, p<0.05$, $\mathrm{n}=7)$ at $5 \mu \mathrm{M}$. Peficitinib also decreased the IL-6 release at $1 \mu \mathrm{M}(24 \%$, $\mathrm{n}=7$ ). In contrast to filgotinib, the JAK-inhibition with peficitinib reduced 